\title{
Elsberg syndrome related to varicella zoster virus infection with painless skin lesions in an elderly woman with poorly controlled type 2 diabetes mellitus
}

This article was published in the following Dove Press journal:

Therapeutics and Clinical Risk Management

\author{
Hitoki Saito' \\ Momoko Ebashi \\ Masaru Kushimoto ${ }^{3}$ \\ Jin Ikeda $^{3}$ \\ Fujiko Egashira' \\ Suguru Yamaguchi' \\ Kentaro Watanabe' \\ Katsuhiko Ogawa ${ }^{2,4}$ \\ Yutaka Suzuki ${ }^{3,4}$ \\ Hisamitsu Ishihara' \\ Midori Fujishiro ${ }^{1,4}$ \\ 'Division of Diabetes and Metabolic \\ Diseases, Nihon University School of \\ Medicine, Tokyo, Japan; ${ }^{2}$ Division of \\ Neurology, Department of Medicine, \\ Nihon University School of Medicine, \\ Tokyo, Japan; ${ }^{3}$ Division of General \\ Medicine, Department of Internal \\ Medicine, Nihon University, Tokyo, \\ Japan; ${ }^{4}$ Department of Internal \\ Medicine, Nihon University Hospital, \\ Tokyo, Japan
}

\begin{abstract}
Patients with diabetes mellitus (DM) are at increased risk of infections, with the urinary tract being the most frequent infection site. Incomplete bladder emptying, frequent urination and abdominal distension are typical symptoms of urinary tract infections (UTIs). A 68-year-old female with a long history of poorly controlled type 2 DM (T2DM) visited our hospital complaining of urinary retention, which was initially diagnosed as cystitis by another doctor. The urologist at our hospital identified a skin rash extending from the left hip to her genital area. A dermatologist was consulted. She was clinically diagnosed with herpes zoster (HZ) involving the left sacral dermatome area. As Elsberg syndrome (ES) was suspected, a lumbar puncture was performed, revealing aseptic meningitis associated with varicella zoster virus (VZV) infection. Intravenous acyclovir with urinary catheterization in combination with methylprednisolone pulse therapy resulted in a good clinical course. HZ very uncommonly involves sacral dermatomes, but it can develop in patients with prolonged poorly controlled DM. Furthermore, early diagnosis can be difficult when patients have diabetic peripheral neuropathy, which may mask symptoms related to skin lesions. Because this disease is potentially severe, detailed examination is important for clinicians managing patients with DM who have complaints indicative of urinary tract disorders.
\end{abstract}

Keywords: herpes zoster, urinary retention, diabetic neuropathy

\section{Introduction}

Elsberg syndrome (ES) is a self-limiting syndrome of acute urinary retention in combination with other, variable, signs of spinal cord dysfunction and cerebrospinal fluid (CSF) pleocytosis. This syndrome was first described by Elsberg in $1913 .{ }^{1}$ Clinicians often do not consider ES in the differential diagnosis of urological emergencies such as acute urinary retention and do not perform appropriate and timely testing for causal viral infections which would allow a definitive diagnosis. Furthermore, several signs of myeloradiculitis are masked by diabetic neuropathy and the diagnosis of ES becomes much more difficult when patients suffer from prolonged poorly controlled diabetes mellitus (DM). Because ES can become severe, an early diagnosis is important. We herein present a case of an elderly woman with DM whose chief complaints were bladder dysfunction and constipation, which led to an early diagnosis of ES associated with varicella zoster virus (VZV) infection.
Correspondence: Midori Fujishiro Division of Diabetes and Metabolic Diseases, Nihon University School of Medicine, 30-I Oyaguchi Kamicho, Itabashi-ku, Tokyo 173-8610, Japan

Tel +8I 339728 III

Fax +8I 339728199

Email fujishiro.midori@nihon-u.ac.jp 


\section{Case report}

A 68-year-old woman, who had been diagnosed with type 2 DM (T2DM), hypertension and dyslipidemia approximately 20 years earlier, was referred to our outpatient department 5 months prior to the present admission. Her HbAlc level was $9.1 \%$, with oral antidiabetic agents (OADs), at the first visit. HbA1c improved to $8.2 \%$ in response to combined therapy with insulin and an OAD after a 3-month educational admission but then had risen again to $8.6 \% 1$ month prior to this admission. She visited a neighborhood doctor complaining of incomplete bladder emptying, frequent urination, constipation and abdominal distension which had started about 10 days earlier. The neighborhood doctor prescribed antibiotics based on a clinical diagnosis of cystitis, but she obtained no symptom relief. She visited the urology department of our hospital for evaluation of severe urinary disturbance and constipation. The urologist performed an abdominal ultrasound and found that urinary retention was due to bladder and rectal disturbance. He also discovered a skin rash extending from the left hip to her genital area and thus consulted a dermatologist working at our hospital (Figure 1). The clinical diagnosis was herpes zoster (HZ) involving the left sacral (S2 and S3) dermatome area, and she was thus admitted to our hospital. On admission, her

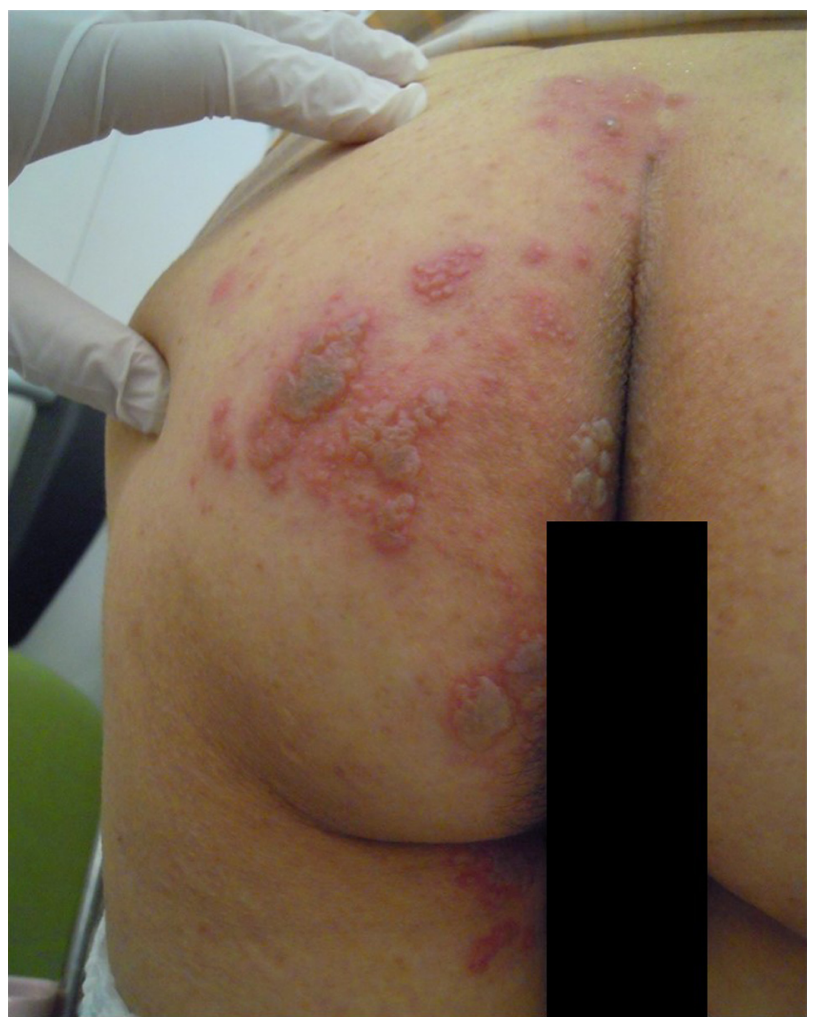

Figure I Multiple clusters of vesicles in a left sacral (S2 and S3) dermatomal pattern, extending from the left hip to the genital area. height was $142 \mathrm{~cm}$ and she weighed $44.6 \mathrm{~kg}$, such that her body mass index (BMI) was 22.1. The vital signs were as follows: body temperature, $37.7^{\circ} \mathrm{C}$; blood pressure, $119 / 74$ $\mathrm{mmHg}$; pulse rate, 117 beats per minute, regular and respiratory rate, 18 per minute. Physical examination revealed no distinct findings or signs of meningeal irritation. Detailed examination revealed multiple clusters of erythematous vesicles arranged in a left sacral (S2 and S3) dermatomal pattern. The patient had experienced only a slight tingling sensation in relation to these lesions and insisted that they were not painful. The physical examination findings were otherwise normal with the exception of an abnormal vibratory sensation in the bilateral lower extremities. Hematological findings were as follows: hematocrit, $41.7 \%$; hemoglobin, $13.7 \mathrm{~g} / \mathrm{dL}$; white blood cells, $7,100 / \mathrm{mm}^{3}$ (neutrophils, $79.0 \%$; eosinophils, $0 \%$; basophils, $0.1 \%$; monocytes, $1.9 \%$; lymphocytes, 19.0\%; no atypical lymphocytes) and platelets, $169,000 / \mathrm{mm}^{3}$. Serum biochemistry, including C-reactive protein (CRP) and urine test results, were normal. The chest $\mathrm{X}$-ray examination, abdominal computed tomography (CT) and brain magnetic resonance imaging (MRI) revealed no abnormalities. Enhanced lumbar MRI detected no disorders possibly responsible for the bladder and rectal disturbance. The laboratory and radiological examinations yielded no findings supporting a diagnosis of vascular disorders, sarcoidosis, malignant lymphoma or other neoplasm. Intramedullary lesions indicating myelitis were not detected on MRI.

Based on the clinical diagnosis of HZ, intravenous acyclovir at a dose of $750 \mathrm{mg}$ every 8 hours was started on admittance with urinary catheterization. In view of the presence of obstructive uropathy and constipation with HZ, suggestive of ES, a lumbar puncture was performed on the 4th day of hospitalization. The CSF was clear without xanthochromia. The CSF cells were recorded as $69 / \mu \mathrm{L}$ (mononuclear cells, $98.6 \%$; polynuclear cells, $1.4 \%$ ), and protein was elevated $(46 \mathrm{mg} / \mathrm{dL})$. The CSF glucose level $(104 \mathrm{mg} / \mathrm{dL})$ was relatively decreased, with a high serum glucose level (319 mg/dL). Gram staining of the CSF was negative for microorganisms. CSF, blood and urine cultures were negative. Aseptic meningitis was therefore diagnosed, the cause of which was confirmed based on a positive CSF PCR reaction for VZV-DNA. The acyclovir dosage was increased to $1.5 \mathrm{~g}$ every 8 hours, which was continuously administered for 14 days, accompanied by methylprednisolone pulse therapy for 3 days. Oral prednisolone was administered and then gradually tapered. Her blood glucose level was controlled with intensive insulin therapy. Severe constipation which had been difficult to control with various combinations of 
several laxatives gradually improved after the 8th hospital day. Urinary retention also showed improvement, and the bladder catheter was removed on the 18th hospital day. A urodynamic study performed on the 21 st hospital day revealed no abnormalities. The VZV infection gradually subsided, and she was discharged on the 33rd hospital day. No neurological disorders associated with cauda equina syndrome have recurred, to date, with more than 6 months of follow-up.

\section{Discussion}

We managed a 68-year-old woman with poorly controlled T2DM, of long duration, whose chief complaints were typical symptoms of urinary tract infection (UTI). The final diagnosis was $\mathrm{HZ}$ presenting as ES. Patients with DM are at increased risk of infections, with the urinary tract being the most common site of infection. ${ }^{2,3}$ Various immune system impairments and incomplete bladder emptying due to autonomic neuropathy may contribute to the pathogenesis of UTI in people with DM. Factors that were found to increase the UTI risk in people with DM included the following: age, metabolic control, long-term complications, primarily diabetic nephropathy and cystopathy as well as the short female urethra located in proximity to the warm, moist, vulvar and perianal areas that are colonized with enteric bacteria. ${ }^{4,5}$ Our patient presented with incomplete bladder emptying, frequent urination and abdominal distension, all of which were consistent with UTI symptoms. Furthermore, she had a complaint of concurrent constipation, which might also be attributable to diabetic autonomic neuropathy. She had no dermatologic complaints but detailed examination revealed skin lesions, leading to referral to a dermatologist and ultimately a diagnosis of $\mathrm{HZ}$. Upon confirmation of the clinical diagnosis, antiviral agents were intravenously administered with urinary catheterization in combination with methylprednisolone pulse therapy. Her subsequent clinical course was good.

ES, also known as lumbosacral meningoradiculitis, is associated with urinary retention and was first reported by Elsberg in 1913. ${ }^{1}$ Human herpes simplex virus (HSV)-2 is reportedly a common etiology of $\mathrm{ES},{ }^{6}$ whereas other herpes viruses, such as HSV-1, VZV, Epstein-Barr virus and cytomegalovirus, have been reported to be associated with lumbosacral radiculomyelitis. ${ }^{7,8}$ Antiviral therapy should be started, with or without steroid therapy, as soon as causative pathogens have been identified, because the symptoms are usually transient though some patients suffer from prolonged urinary disturbance. ${ }^{9}$ According to the diagnostic criteria for ES, with the five levels of diagnostic certainty suggested by
Savoldi et al, ${ }^{8}$ our patient fits into the "laboratory-supported definite" category that requires proven HSV infection. Thus, our patient was treated with a combination of intravenous antiviral acyclovir and steroid pulse therapy and discharged without sequelae. $\mathrm{HZ}$ is an infectious vesicular skin rash causing pain with a dermatomal distribution which occurs in response to reactivation of a previous VZV infection. The incidence of $\mathrm{HZ}$ and the severity of postinfection complications increase with age. Several recent studies have suggested that DM represents an important risk factor for $\mathrm{HZ}$ and increases the severity of the clinical course of $\mathrm{HZ}$, such that efforts to increase zoster vaccination have been advocated. ${ }^{10,11} \mathrm{HZ}$ very uncommonly involves sacral dermatomes, occasionally making early diagnosis difficult when patients have diabetic peripheral neuropathy. Our patient, who had no complaints of pain associated with her skin lesions, illustrates this challenge. Because this disease can progress and take a severe course, ${ }^{12,13}$ careful examination is essential for clinicians managing people with DM who have symptoms suggestive of UTI.

\section{Conclusion}

ES related to VZV should be considered in the differential diagnosis of patients with prolonged poorly controlled T2DM who have complaints of urinary disorder, even in the absence of dermatologic complaints. Because progression to a severe disease state occurs in some patients, with high risk of sequelae, keeping this disease in mind while performing a thorough examination is essential.

\section{Abbreviations}

BMI, body mass index; CT, computed tomography; DM, diabetes mellitus; ES, Elsberg syndrome; HSV, herpes simplex virus; CRP, C-reactive protein; MRI, magnetic resonance imaging; OAD, oral antidiabetic agents; UTI, urinary tract infections; CSF, cerebrospinal fluid; VZV, varicella zoster virus; HZ, herpes zoster; T2DM, type 2 diabetes mellitus.

\section{Disclosure}

The authors report no conflicts of interest in this work.

\section{References}

1. Elsberg C. Experiences in spinal surgery. Observations upon 60 laminectomies for spinal disease. Surg Gynecol Obstet. 1913;16:117-132.

2. Shah BR, Hux JE. Quantifying the risk of infectious diseases for people with diabetes. Diabetes Care. 2003;26(2):510-513.

3. Joshi N, Caputo GM, Weitekamp MR, Karchmer AW. Infections in patients with diabetes mellitus. $N$ Engl J Med. 1999;341(25): 1906-1912. 
4. Nitzan O, Elias M, Chazan B, Saliba W. Urinary tract infections in patients with type 2 diabetes mellitus: review of prevalence, diagnosis, and management. Diab Metab Syndr Obes. 2015;8:129-136.

5. He K, Hu Y, Shi JC, Zhu YQ, Mao XM. Prevalence, risk factors and microorganisms of urinary tract infections in patients with type 2 diabetes mellitus: a retrospective study in China. Ther Clin Risk Manag. 2018;14:403-408.

6. Eberhardt O, Küker W, Dichgans J, Weller M. HSV-2 sacral radiculitis (Elsberg syndrome). Neurology. 2004;63(4):758-759.

7. Küker W, Schaade L, Ritter K, Nacimiento W. MRI follow-up of herpes simplex virus (type 1) radiculomyelitis. Neurology. 1999;52(5): 1102-1103.

8. Savoldi F, Kaufmann TJ, Flanagan EP, Toledano M, Weinshenker BG, $\mathrm{Bg}$ W. Elsberg syndrome: A rarely recognized cause of cauda equina syndrome and lower thoracic myelitis. Neurol Neuroimmunol Neuroinflamm. 2017;4(4):e355.
9. Hamai K, Takaoka Y, Kojima A, Nomura T, Matsui M. Two cases of Herpes Zoster Accompanied by Bladder/rectum Disorder. Skin Res. 2014; 13:93-96.

10. Kawai K, Yawn BP. Risk Factors for Herpes Zoster: A Systematic Review and Meta-analysis. Mayo Clin Proc. 2017;92(12):1806-1821.

11. Papagianni M, Metallidis S, Tziomalos K. Herpes Zoster and Diabetes Mellitus: A Review. Diabetes Ther. 2018;9(2):545-550.

12. Matsumoto H, Shimizu T, Tokushige S, Mizuno H, Igeta Y, Hashida H. Rectal ulcer in a patient with VZV sacral meningoradiculitis (Elsberg syndrome). Intern Med. 2012;51(6):651-654.

13. Malkud S, Patil S. Disseminated Cutaneous Herpes Zoster in a Patient with Uncontrolled Diabetes Mellitus. J Clin Diagn Res. 2015;9:WD01-WD02.

\section{Publish your work in this journal}

Therapeutics and Clinical Risk Management is an international, peerreviewed journal of clinical therapeutics and risk management, focusing on concise rapid reporting of clinical studies in all therapeutic areas, outcomes, safety, and programs for the effective, safe, and sustained use of medicines. This journal is indexed on PubMed Central, CAS,
EMBase, Scopus and the Elsevier Bibliographic databases. The manuscript management system is completely online and includes a very quick and fair peer-review system, which is all easy to use. Visit http://www.dovepress.com/testimonials.php to read real quotes from published authors. 